\title{
Radiotherapy as an effective treatment modality for follicular lymphoma: a single institution experience
}

\author{
Seo Hee Choi, MD', Jaeho Cho, MD, PhD'1, Jin Seok Kim, MD, PhD², \\ June-Won Cheong, MD, PhD², Chang-OK Suh, MD, PhD' \\ 1 Department of Radiation Oncology, Yonsei University College of Medicine, Seoul; \\ ${ }^{2}$ Division of Hematology, Department of Internal Medicine, Yonsei University College of Medicine, Seoul, Korea
}

\begin{abstract}
Purpose: Follicular lymphoma (FL) is an indolent non-Hodgkin's lymphoma that is highly sensitive to radiotherapy (RT). However, the effectiveness of RT has not been well established. We reviewed our experiences to assess the role of RT for FL and analyze treatment results.

Materials and Methods: Retrospective analysis was done on 29 patients who received first RT between January 2003 and August 2013. Of 23 early stage (stage I, II) patients, 16 received RT alone, four received chemotherapy followed by RT, two received RT postoperatively, and one received salvage RT for relapse after resection. Six advanced-stage (stage III, IV) patients received RT after chemotherapy: two received consolidation RT, three received salvage RT for residual lesions, and one received RT for progressive sites. Median RT dose was 30.6 Gy (range, 21.6 to 48.6 Gy). Median follow-up duration was 62 months (range, 6 to 141 months).

Results: All patients showed complete response in the radiation field. Eight outfield relapses were reported. Seven patients received salvage treatment (three chemotherapy, four RT). Four patients showed excellent responses, especially to RT. Estimated 5-year and 10-year relapse-free survivals were 72\% and 60\%. In the RT-alone group, 5-year relapse-free survival was 74.5\%. All advanced-stage patients were disease-free with 100\% 5-year overall survival. Disease-specific death was noted in only one patient; four others died of other unrelated causes. No significant toxicity was reported.

Conclusion: RT resulted in excellent treatment outcomes for all FL stages when used as a primary treatment modality for early stage or salvage-treatment modality for advanced-stage disease.
\end{abstract}

Keywords: Follicular lymphoma, Radiotherapy, Treatment outcome

\section{Introduction}

Follicular lymphoma (FL) is a tumor of follicle center B cells (centrocytes and centroblasts) and has a follicular (nodular) pattern that is morphologically similar to normal germinal centers. FL is approximately $20 \%$ of all newly diagnosed lymphoma cases and is reported to be the second most common subtype of non-Hodgkin lymphoma in the United States. FL is relatively rare in Korea; at $2.9 \%$, it is the sixth most common non-Hodgkin lymphoma subtype in Korea [1]. For early stage FLs, the current treatment options in patients with stage I or contiguous stage II FL include observation, radiotherapy (RT), chemotherapy, and combined treatment modalities of chemotherapy plus RT. For advanced-stage FLs,

Received 10 June 2015, Revised 13 July 2015, Accepted 3 August 2015.

Correspondence: Chang-Ok Suh, MD, PhD, Department of Radiation Oncology, Yonsei University College of Medicine, 50 Yonsei-ro, Seodaemun-gu, Seoul 03722, Korea. Tel: +82-2-2228-8117, Fax: +82-2-2227-7823, E-mail: cosuh317@yuhs.ac

(c) This is an Open Access article distributed under the terms of the Creative Commons Attribution Non-Commercial License (http://creativecommons.org/ licenses/by-nc/4.0/) which permits unrestricted non-commercial use, distribution, and reproduction in any medium, provided the original work is properly cited.

www.e-roj.org 
treatment is required in symptomatic or bulky tumors, and chemotherapy including rituximab is usually recommended.

Localized and early stage FLs (Ann Arbor stage I and II) are 22\%-33\% of all FL cases [2]. RT alone is the wellrecognized, preferred treatment of choice in several international guidelines $[3,4]$. FL has long been recognized as more radiosensitive than other lymphoma subtypes and the efficacy of RT is observed even at low radiation doses. Several studies reviewed the long-term outcomes of RT alone and reported relapse-free survival (RFS) of 50\% at 10 years and approximately $40 \%$ at 15 years; overall survival (OS) rates were 60\%-80\% and 35\%-45\%, respectively [5]. Thus, RT is regarded as a curative treatment modality for a significant number of patients with early stage $\mathrm{FL}$.

For advanced-stage $F L$, a watch-and-wait policy is the preferred treatment of choice for patients without symptoms and with low tumor burden. Because of the indolent course of $\mathrm{FL}$ and the lack of life-prolonging or curative therapy, this strategy was established in the 1990s. A few prospective randomized studies demonstrated that systemic therapy could be safely delayed until treatment is necessary without negatively influencing patient outcomes $[6,7]$. For patients with a high tumor volume or symptoms requiring treatment, a combination of rituximab and chemotherapy is the standard treatment, with higher response and survival rates than chemotherapy without rituximab [8-12]. RT could be a good alternative approach in these patients. However, because of the lack of data on RT as a first-line treatment for advanced-stage $F L, R T$ is currently recommended only for palliative treatment of locally symptomatic disease or second-line therapy for relapsed or refractory disease. Several studies have found RT to be an excellent palliative modality that provides longterm local control and clinical benefits even for advancedstage FL. Even very small doses of $4 \mathrm{~Gy}$ could have benefits for patients with advanced, relapsed, or refractory stage $\mathrm{FL}[13,14]$. With the extreme radiosensitivity of $F L, R T$ for advancedstage FL should also be established and is expected to be more extensively used than under currently recommended use. No studies have investigated RT outcomes in advanced-stage FL patients, especially in Korea.

In Korea, most radiation oncologists do not have opportunity to experience treating $\mathrm{FL}$ because of the low incidence and low RT referral rates. Therefore, we retrospectively reviewed RT experience at our institution with the aim of analyzing overall treatment results and defining the role of $\mathrm{RT}$ for $\mathrm{FL}$ treatment.

\section{Materials and Methods}

\section{Patients}

A total of 34 patients with FL who were diagnosed and received first RT between September 2000 and August 2013 at Severance Hospital were identified. Two patients with uncertain pathology and three with a short follow-up period were excluded. Therefore, a total of 29 patients were included in the current study. All patients were pathologically diagnosed with FL by biopsy or surgical excision. Ann Arbor stage I and II FL was classified as early stage, and Ann Arbor stage III and IV was classified as advanced-stage. Patients were staged according to physical examination, imaging studies, blood chemistry, and bone marrow biopsy. Imaging studies included computed tomography (CT) scan or positron emission tomography (PET)-CT. Nine patients were diagnosed using CT scan only (June 2000 to January 2003) and 20 patients were diagnosed using both CT and PET-CT (June 2003 to June 2013). Complete blood counts and lactate dehydrogenase (LDH) levels were determined. Revised Follicular Lymphoma International Prognostic Index 2 (FLIPI 2) scores were calculated based on individual parameters (low risk group, $0-1$; intermediate risk group, 2; high risk group, 3-5) [15].

The early stage group had 23 patients (Ann Arbor stage I, 17; stage II, 6); the advanced-stage group had 6 (Ann Arbor stage IIIA, 2; stage IVA, 4). Median age at diagnosis was 59 years (range, 16 to 83 years). Median follow-up duration was 62 months (range, 9 to 155 months) after initial diagnosis. Patient and tumor characteristics are in Table 1.

\section{Treatment}

Of the 23 early stage patients, 16 received RT alone, four received chemotherapy followed by $\mathrm{RT}$, two received $\mathrm{RT}$ postoperatively, and one received salvage RT for relapse after resection. All six advanced-stage patients received RT after chemotherapy: two received consolidation $\mathrm{RT}$, three received salvage RT for residual lesions, and one received RT for progressive sites (Fig. 1).

Treatment details of early stage patients were as follows. In the RT-alone group ( $n=16), 4$ patients received extendedfield RT (EFRT) (three as supradiaphragmatic extended-field $R T$, mantle field with or without Waldeyer's ring, and one as subdiaphragmatic extended-field RT [inverted $Y$ field]) and 12 received involved-filed RT (IFRT). The median total RT dose was 30.6 Gy (range, 24 to $45 \mathrm{~Gy}$ ) as $1.8 \mathrm{~Gy}$ or $2.0 \mathrm{~Gy}$ per fraction. In the RT-after-chemotherapy group $(n=4)$, three patients refused additional chemotherapy after receiving 
Table 1. Patient characteristics $(n=29)$

\begin{tabular}{|c|c|}
\hline Variable & No. $(\%)$ \\
\hline Age (yr), median (range) & $58(16-83)$ \\
\hline$\geq 60$ & $14(48.3)$ \\
\hline$<60$ & $15(51.7)$ \\
\hline \multicolumn{2}{|l|}{ Sex } \\
\hline Male & $11(37.9)$ \\
\hline Female & $18(62.1)$ \\
\hline \multicolumn{2}{|c|}{ No. of involved nodal areas } \\
\hline$>4$ & $7(24.1)$ \\
\hline$\leq 4$ & $22(75.9)$ \\
\hline \multicolumn{2}{|l|}{ BM involvement } \\
\hline Yes & $2(93.1)$ \\
\hline No & $27(6.9)$ \\
\hline \multicolumn{2}{|l|}{ Anemia $^{\text {a) }}$} \\
\hline Yes & 6 (79.3) \\
\hline No & $23(20.7)$ \\
\hline \multicolumn{2}{|l|}{$\mathrm{LN}>6 \mathrm{~cm}$} \\
\hline Yes & $2(93.1)$ \\
\hline No & $27(6.9)$ \\
\hline \multicolumn{2}{|l|}{ LDH elevation ${ }^{b)}$} \\
\hline Yes & $15(51.7)$ \\
\hline No & $13(44.8)$ \\
\hline No information & $1(3.5)$ \\
\hline \multicolumn{2}{|c|}{$\beta 2$-microglobulin elevation ${ }^{c}$} \\
\hline Yes & $7(24.1)$ \\
\hline No & $17(58.6)$ \\
\hline No information & 5 (17.3) \\
\hline \multicolumn{2}{|l|}{ Ann Arbor stage } \\
\hline IA & $13(44.8)$ \\
\hline IEA & $3(10.3)$ \\
\hline IB & $1(3.5)$ \\
\hline$\| A$ & $5(17.2)$ \\
\hline IIEA & $1(3.5)$ \\
\hline$\| \mathrm{A}$ & $2(6.9)$ \\
\hline IVA & $4(13.8)$ \\
\hline \multicolumn{2}{|l|}{ FLIPI2 group } \\
\hline Low risk & $15(51.7)$ \\
\hline Intermediate risk & $7(24.1)$ \\
\hline High risk & $4(13.8)$ \\
\hline Unevaluable & $3(10.4)$ \\
\hline
\end{tabular}

$B M$, bone marrow; LN, lymph node; LDH, lactate dehydrogenase; FLIPI2, Follicular Lymphoma International Prognostic Index 2.

${ }^{a)}$ Anemia, $<13 \mathrm{~g} / \mathrm{dL}$ (male) or $12 \mathrm{~g} / \mathrm{dL}$ (female). ${ }^{b} \mathrm{LDH}$ elevation, $>247 \mathrm{IU} / \mathrm{L}$. ${ }^{\mathrm{c}} \beta 2$-microglobulin elevation, $>2.74 \mathrm{mg} / \mathrm{L}$.

1-2 chemotherapy cycles and one received RT for suspected residual disease after 6 chemotherapy cycles. In the RT-aftersurgery group $(n=2)$, all patients were initially diagnosed with suspicious parotid gland masses and first received surgical excision with a curative or diagnostic aim. After pathological examination, additional RT was performed postoperatively to cover microscopically suspicious areas around the operation site (All patients were treated with IFRT with $21.6 \mathrm{~Gy}$ in $1.8 \mathrm{~Gy}$ fractions or $40 \mathrm{~Gy}$ in $2 \mathrm{~Gy}$-fractions). In the salvage-RT group (n $=1$ ), the patient was diagnosed with a suspicious lung nodule in the left lower lobe. After surgical excision, pathological diagnosis confirmed FL. After 2 years of follow-up without adjuvant treatment, a relapse in the mediastinal lymph node area was discovered and $30 \mathrm{~Gy}$ of mantle field RT including the mediastinal lymph node area was selected as the salvage treatment.

Treatment details for advanced-stage patients were as follows. In the salvage-RT group, three patients received RT for residual lesions after chemotherapy completion and one patient received RT for progressive disease sites. Almost all patients received 30.6 to $35 \mathrm{~Gy}$ of extended-field RT (two with additional whole-abdomen RT of 24 Gy), except for a single patient who received IFRT $30.6 \mathrm{~Gy}$. Of the consolidation-RT patients, one was initially diagnosed with $\mathrm{FL}$ involving the left neck level $\mathrm{V}$ lymph node and left proximal humerus. This patient received 6 cycles of rituximab plus cyclophosphamide, doxorubicin, vincristine, and prednisone (R-CHOP) chemotherapy; complete remission (CR) at disease sites was noted. As a consolidation aim, an additional 25.2 Gy IFRT was administered to the left neck and humerus. Another patient was initially diagnosed with $\mathrm{FL}$ involving the neck, axilla, para-aortic, and inguinal lymph node areas; this patient received with 6 cycles of CAVOP (cyclophosphamide, adriamycin, vincristine, VP-16, prednisone chemotherapy). After $\mathrm{CR}$, an additional $24 \mathrm{~Gy}$ of extended-field RT (mantle field) was administered.

\section{Response evaluation and statistical analysis}

Response to therapy was evaluated within 6-12 weeks after RT and careful long-term follow-up evaluations were maintained. Regular posttreatment monitoring was based on laboratory tests and clinical or radiological examination if appropriate of all peripheral lymph node sites. Follow-up imaging studies were at least a CT scan of the involved region with PETCT scans used more recently. CR was defined as complete disappearance of all detectable evidence of disease. Responses after the initial treatment schedule were evaluated in all patients and relapse or progression, defined as appearance of a new lesion or increase in initial tumor size, was evaluated with follow-up images. Toxicity was graded using the Common Terminology Criteria for Adverse Events (CTCAE) ver. 4.0.

OS was estimated from initial diagnosis to last follow-up or date of death from any cause. RFS was measured from initial diagnosis to date of relapse/progression or to last follow- 
A

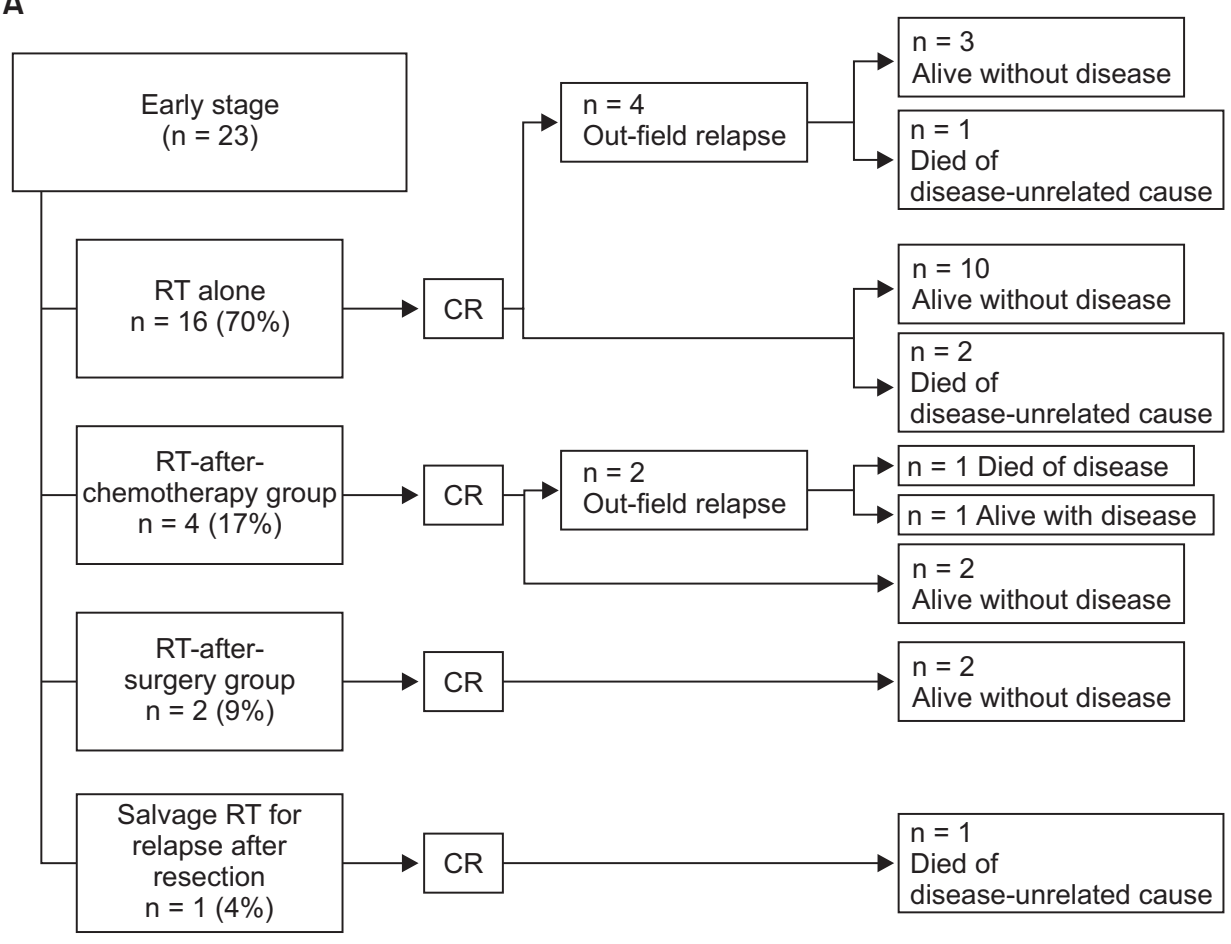

B

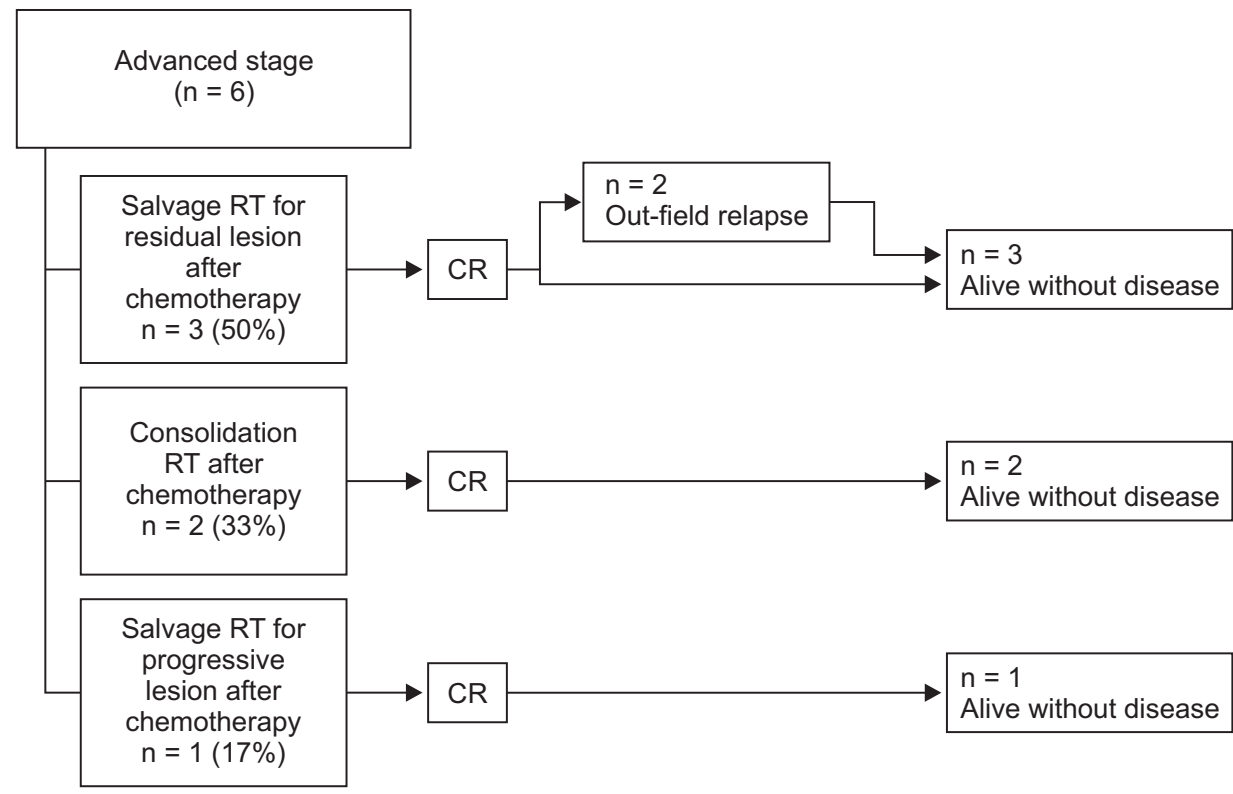

Fig. 1. Treatment flowchart of 29 patients with follicular lymphoma. $R T$, radiotherapy; $C R$, complete remission.

up date for patients who did not experience these events. OS and RFS were calculated using the Kaplan-Meier method. The statistical significance of differences in Kaplan-Meier estimates was assessed using the log-rank test. All point estimates were accompanied by $95 \%$ confidence interval. A $p<0.05$ was considered statistically significant. Statistical analysis was performed using IBM SPSS ver. 20 (IBM, Armonk, NY, USA).

\section{Results}

\section{Early stage patients}

After RT, all patients showed CR in the radiation field. In the RT-alone group ( $n=16$ ), all patients $(100 \%)$ showed CR in radiation field. There is no infield relapse, and four patients (one with mantle field, three with IFRT) showed outfield relapse (median RFS from initial diagnosis, 34 months; range, 11 to 
84 months). Of these patients, two were treated with salvage IFRT at relapse sites and two received salvage chemotherapy. Three patients (19\%) died, and all deaths were unrelated to FL. Two patients, aged 75 and 90 years, died of other underlying medical diseases and one died of a second malignancy 122 months after FL diagnosis.

In the RT-after-chemotherapy group $(n=4)$, all patients (100\%) showed CR in radiation field. Two patients showed outfield relapse at 17 and 43 months after RT. One initially responded well to salvage treatment with several cycles of chemotherapy, but infield disease relapse in the inguinal and pelvic lymph node area continued. This patient was enrolled in a clinical trial and was alive with disease and receiving chemotherapy. The other patient did not respond to salvage treatment despite several cycles of RT and chemotherapy, and died of disease 2 years after systemic relapse. All patients except this patient (75\%) are alive until last follow-up date.

Both RT-after-surgery group and salvage RT for relapse after resection group also showed $100 \%$ of CR in radiation field. There was only one death in salvage-RT group, which was unrelated to FL (advanced gastric cancer). There was no treatment-related toxicity after RT in all patients of early stage group to the last follow-up date.

\section{Advanced-stage patients}

All six advanced-stage patients received RT after chemotherapy. Two received consolidation $R T$, three received salvage $R T$ for residual lesions, and one received $\mathrm{RT}$ for progressive sites. After $\mathrm{RT}$, all patients showed $\mathrm{CR}$ in the radiation field.

In the salvage-RT group, all patients (100\%) showed CR in radiation field, and there was no disease-related death.
Two of a total of four patients showed relapses at 22 and 26 months after RT. One showed suspected outfield relapse in the left para-aortic lymph node at 29 months after initial diagnosis. This patient was alive with stable disease without additional salvage treatment. Another patient was a 42-yearold woman who was initially diagnosed with Ann Arbor stage IIIA FL in the bilateral supraclavicular fossa, subcarinal, right axilla, abdominal, bilateral iliac, and bilateral inguinal lymph nodes. She initially received 6 cycles of CHOP chemotherapy and interferon-alpha therapy but after 1.5 months, multiple residual lesions in the subcarinal, left iliac lymph node chain were confirmed on follow-up images. Whole abdomen RT of $24 \mathrm{~Gy}$ in 16 fractions and mantle field RT of $30 \mathrm{~Gy}$ in 20 fractions were administered. After whole abdomen $R T$, an additional radiation dose up to $34.5 \mathrm{~Gy}$ was added to the paraaortic, pelvic lymph node area. After 6 months, CR at treated sites was seen by PET-CT; this response continued until March 2005. However, outfield relapses in the left neck level II and right axilla lymph node area were detected 33 months after diagnosis. Additional IFRT was given as salvage treatment, specifically to the Waldeyer's ring to the upper neck lymph node at $30.6 \mathrm{~Gy}$ in 17 fractions and to the right axilla lymph node at 30.6 Gy in 17 fractions with additional radiation in the left neck level II lymph node up to $36 \mathrm{~Gy}$. This patient was alive without disease 152 months after diagnosis. All patients including these relapsed patients are alive and disease-free without any treatment-related complications.

In consolidation RT group and salvage RT for progressive lesion after chemotherapy group, 100\% of CR was also noted and there was no relapse or disease-related death. Two patients who received consolidation RT after chemotherapy are

B

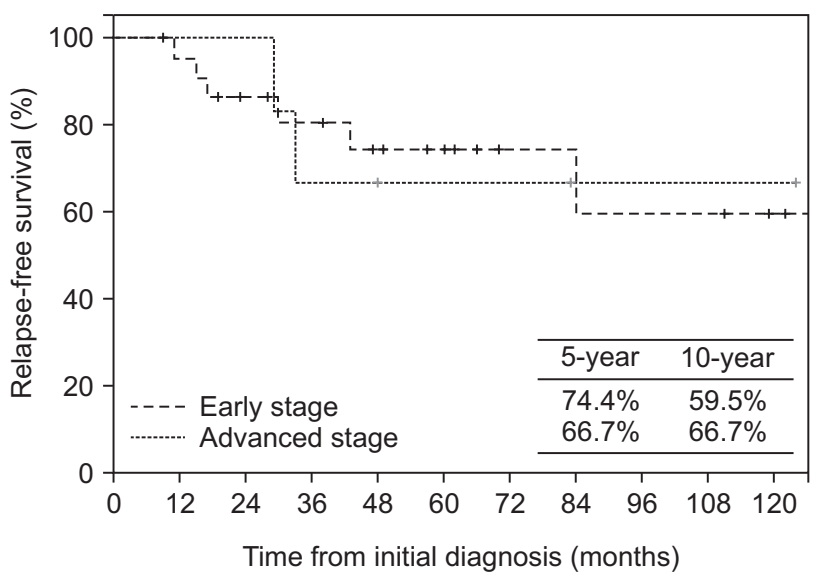

Fig. 2. (A) Overall survival and (B) relapse-free survival from initial diagnosis. 
alive without evidence of disease at 75 and 118 months after RT. One patient who received salvage RT for progressive lesion is also alive without disease at 23 months after RT. There was no treatment-related toxicity after RT in all advanced-stage group patients to the last follow-up date.

\section{Survival}

From initial diagnosis, estimated 5-year RFS was 72\% and 10year RFS was 60\%. In early stage patients, 5-year RFS was 74.4\% and 10-year RFS was 59.5\%. In the RT-alone group, 5-year RFS was 74.5\%. In advanced-stage patients, 5-year RFS was $66.7 \%$ and 10 -year RFS was 66.7\%. From the date of RT start, overall 5-year RFS was 71.8\% and 10-year RFS was $61.6 \%$. In early stage patients, 5-year RFS was $74.4 \%$ and $10-$ year RFS was 59.5\%. In advanced-stage patients, 5-year RFS was 62.5\% and 10-year RFS was 62.5\% (Fig. 2).

From initial diagnosis, 5-year OS was 100\% and at 10-year OS was $65.2 \%$. OS from the date of RT start was $100 \%$ at 5 years and $54.9 \%$ at 10 years. A total of five deaths were noted. Disease-specific death occurred in one early stage patient; another four died of second malignancies at RT-unrelated sites or of underlying disease. For early stage patients, 5-year OS was 100\% and 10-year OS was 54.3\%. For advanced-stage patients, both 5 -year and 10 -year OS were 100\%. From the date of RT start, for early stage patients, 5-year OS was 100\% and 10-year OS 41.9\%; for advanced-stage patients, 5-year and 10-year OS were 100\% (Fig. 2).

Age older than 60 years had a significant impact on both RFS and OS in univariate analysis ( $p=0.013$ and $p=0.056$, respectively). In patients younger than 60 years old, 5-year OS was 100\% and 5-year RFS was 57.8\%; 10-year OS was $88.9 \%$ and 10 -year RFS was 38.5\%. Patients older than 60 years old showed worse prognosis (5-year OS 100\% and RFS 92.3\%; 10-year OS 55.6\% and RFS 92.3\%). Analysis of FLIPI2 scores showed that only RFS between the intermediate and high grade groups was significantly different and other comparisons were insignificant.

In relapsed patients, no significant relationship was demonstrated between treatment characteristics and response rates; patients with relapsed disease were very responsive to RT. Overall, 50\% patients were alive without disease with various salvage treatments (median OS from diagnosis, 95 months), with one disease-related death reported. Characteristics of patients who relapsed after RT are in Table 2.

\section{Discussion and Conclusion}

In this retrospective study, RT as a primary treatment modality (RT alone) for early stage patients or a salvage treatment modality for advanced-stage patients resulted in excellent treatment outcomes for patient with all stages of FL. All patients who received RT as a first-line or secondline treatment showed $C R$ in the radiation field and excellent RFS and OS. We confirmed that FL is a highly radiosensitive malignancy and RT could be a treatment option comparable to chemotherapy even including rituximab.

In previous studies, FL prognosis and treatment options were reported as heterogeneous. Prognostic factors in patients with $\mathrm{FL}$ are diverse and include age, stage, tumor burden, bone marrow involvement, systemic symptoms, performance status, serum LDH, hemoglobin, erythrocyte sedimentation rate, and $\beta 2$-microglobulin [16-21]. In 2004, after a large international cooperative effort, the FLIPI score was established [20]. However, this score was designed before the era of rituximab and the initial study cohort does not represent the present disease course. Since the FLIPI score has several other limitations, the International Follicular Lymphoma Prognostic Factor Project group proposed the FLIPI2 score. Results with the FLIPI2 score show that $\beta 2$-microglobulin higher than the upper limit of normal, longest diameter of the largest involved node longer than $6 \mathrm{~cm}$, bone marrow involvement, hemoglobin level lower than $12 \mathrm{~g} / \mathrm{dL}$, and age older than 60 years are independently predictive factors for RFS; these factors are proposed for risk group classification [15]. However, in our study, only age older than 60 years was a significant prognostic factor for all FL stages. FLIPI2 score was not well correlated with prognosis. These results might be due to the small number of cohort patients.

Many patients are diagnosed with advanced-stage $\mathrm{FL}$ and 15\%-30\% present with localized or early stage disease. Treatment of early stage FL remains heterogeneous and controversial because of a lack of randomized phase III trials in this subgroup. According to many retrospective studies, RT is considered the standard of care at this stage. Alternative treatments include observation, chemotherapy, immunotherapy, or both, with or without RT. Previous studies on RT outcomes demonstrated excellent local tumor control (>90\%) and reported RFS in the range of 50\% at 10 years and approximately $40 \%$ at 15 years. OS rates were $60 \%$ to $80 \%$ at 10 years and $35 \%$ to $45 \%$ at 15 years [5,22-24]. Chemotherapy alone or combined therapy shows similar response rates for early stage $F L$, but no definitive data from other studies 


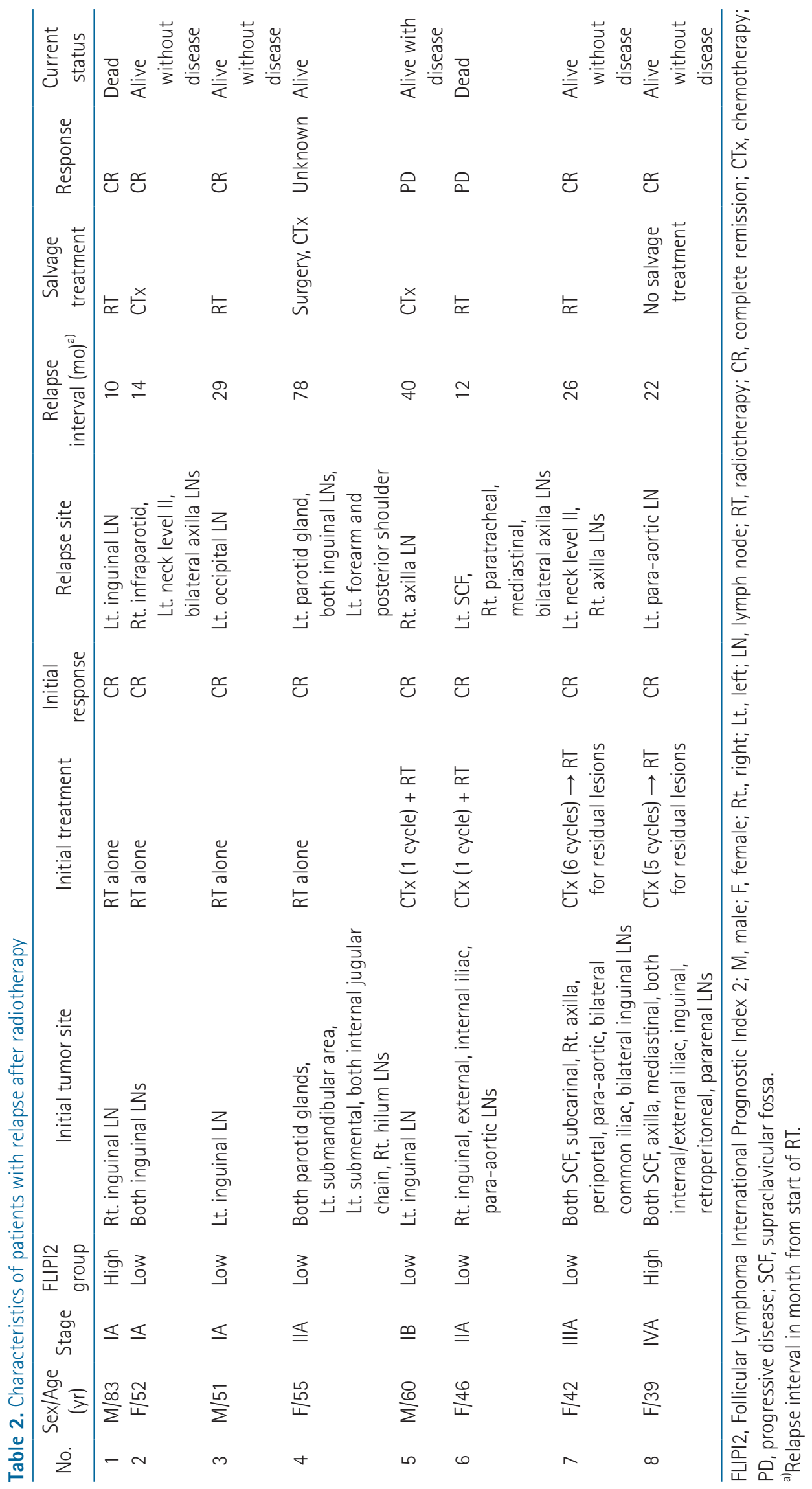


demonstrated an outstanding survival advantage. Despite being considered a standard treatment, RT for early stage $\mathrm{FL}$ is greatly underused in both the United States and Korea. The reasons for this underuse could be low accessibility for RT, lack of awareness about RT by hematologists, and concerns about radiation toxicity. However, FL is known to be highly sensitive to radiation and even very low doses may be adequate for extended local control. In our retrospective results, the RTalone group showed excellent survival outcomes with low doses and small fields, comparable to previously reported retrospective or prospective outcomes for both RT-alone and combined therapy groups. A lower dose approach would make RT much safer and easier to administer than chemotherapy with comparable treatment outcomes. Because of these advantages, RT should be considered more frequently for early stage FL.

For advanced-stage $\mathrm{FL}$, the addition of rituximab to standard chemotherapy is recommended for first-line treatment in symptomatic patients. Both expectant management (watchful waiting) and rituximab monotherapy are recommended for patients without symptoms or with low tumor burden according to several international guidelines. The benefits of rituximab have been shown for rituximab followed by rituximab maintenance or in combination with other chemotherapy regimens. Rituximab followed by rituximab maintenance has overall response rates of $76 \%$ and a median RFS of 34 months in symptomatic patients [25]. Rituximab with other chemotherapy improves both response rates and OS rates according to four randomized studies showing response rates of $81 \%$ to $96 \%$ and median remission durations of 27 to 66 months $[8,10,12,26]$. R-CHOP was the most effective treatment option with better response rates (91\%-93\%), RFS (3-year, 68\%; median, 40.9 months) and lower toxicity than other combination options [27-29].

Limited studies are available on RT as a first-line treatment for advanced-stage FL. Studies that showed survival benefits were performed with palliative intent for symptomatic or some bulky diseases. In the rituximab era, much fewer reports on RT alone have been published due to the outstanding response rates of rituximab. Thus, RT is currently recommended only for patients with symptomatic disease as a palliative treatment option. Response rates range from 75\% to 92\% and $\mathrm{CR}$ rates range from 36\% to $61 \%$ for advanced-stage $\mathrm{FL}$. In several previous retrospective studies on central lymphatic irradiation including mantle field, whole abdomen, and pelvis fields, including in patients with stage III disease, 5-year RFS rates fell to about 50\% in most series $[23,30]$. McClanahan et al. [31] showed the benefit of consolidation IFRT after immunochemotherapy including rituximab was evaluated in patients in the HD2000 prospective trial. Although no significant difference was noted between exposure to IFRT and likelihood of relapse $(p=0.751)$ or at a specific location ( $p=0.66), 6$-year RFS was $52 \%$ and 6-year OS was $80 \%$ after IFRT; RFS was $48 \%$ and OS was $73 \%$ without IFRT. In our study, although only small numbers of advanced-stage patients were included, 5-year RFS from initial diagnosis was $66.7 \%$ and 5-year OS from initial diagnosis was 100\%, which were similar to previous reports. We found no differences in treatment outcomes for RT for bulky diseases. Asserting that RT alone as a first-line treatment option for advanced-stage patients might not be feasible, because of the small number of reports showing benefits in heterogeneous patient groups in the rituximab era. However, RT can be a reasonable treatment option with excellent response rates and very low toxicity for relapsed or refractory patients. Treatment options for relapsed FL vary and include chemotherapy with or without rituximab, rituximab monotherapy, local $\mathrm{RT}$, and radioimmunotherapy. Previous treatment history and duration of response should be considered in selecting the most appropriate salvage treatment. Until now, CHOP chemotherapy or rituximab have also been reasonable options, but they have not been evaluated prospectively and have marginal utility. Our results demonstrated that RT was a very effective salvage treatment option for relapsed patients.

Our study was limited, first because of the retrospective study design and small sample size. However, few studies have reported RT outcomes for FL because of the low incidence in Korea and treatment options other than RT. Especially for patients with advanced-stage disease, most institutions regard chemotherapy including rituximab as a first-line treatment and RT experiences have not been widely shared between institutions. Thus, our report is valuable because it addresses this situation. Second, our study cohort included only patients who were referred to our radiation oncology department. In particular, the advanced-stage group had a small number of patients with varying disease status. Although physicians were concerned about radiation toxicity in selecting treatment modalities, all patients tolerated RT without serious complications. Because recent recommendations about RT dose and fields have become much lower and smaller (IFRT, ISRT), RT is expected to be more comfortable for patients without compromising treatment outcome. Some good responders in our advanced-stage group were older than 50 year-old or had severe disease status despite several cycles of 
chemotherapy. Thus, we concluded from our results that RT was highly effective regardless of the patient's performance status, disease severity, or disease sites.

In conclusion, our experience showed that RT can be a highly effective treatment modality as a primary or salvage treatment option for management of all stages of FL. In Korea, very few studies have assessed use of RT for non-Hodgkin lymphoma, especially FL. Our findings will therefore be valuable for selecting the most appropriate treatment options for FL. RT can be more widely recommended for patients with all stages of $\mathrm{FL}$, with or without chemotherapy.

\section{Conflict of Interest}

No potential conflict of interest relevant to this article was reported.

\section{References}

1. Kim JM, Ko YH, Lee SS, et al. WHO classification of malignant Iymphomas in Korea: report of the third Nationwide Study. Korean J Pathol 2011;45:254-60.

2. Friedberg JW, Byrtek $M$, Link BK, et al. Effectiveness of firstline management strategies for stage I follicular lymphoma: analysis of the National LymphoCare Study. J Clin Oncol 2012; 30:3368-75.

3. Zelenetz AD, Gordon LI, Wierda WG, et al. Non-Hodgkin's Lymphomas, Version 2.2014. J Natl Compr Canc Netw 2014;12: 916-46.

4. Ghielmini M, Vitolo U, Kimby E, et al. ESMO Guidelines consensus conference on malignant lymphoma 2011 part 1 : diffuse large B-cell lymphoma (DLBCL), follicular lymphoma (FL) and chronic lymphocytic leukemia (CLL). Ann Oncol 2013; 24:561-76.

5. Guadagnolo BA, Li S, Neuberg D, et al. Long-term outcome and mortality trends in early-stage, Grade 1-2 follicular lymphoma treated with radiation therapy. Int J Radiat Oncol Biol Phys 2006;64:928-34.

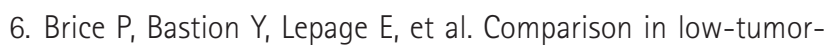
burden follicular lymphomas between an initial no-treatment policy, prednimustine, or interferon alfa: a randomized study from the Groupe d'Etude des Lymphomes Folliculaires. Groupe d'Etude des Lymphomes de I'Adulte. J Clin Oncol 1997;15:11107.

7. Ardeshna $K M$, Smith $P$, Norton $A$, et al. Long-term effect of a watch and wait policy versus immediate systemic treatment for asymptomatic advanced-stage non-Hodgkin lymphoma: a randomised controlled trial. Lancet 2003;362:516-22.

8. Hiddemann $W_{1}$ Kneba $M$, Dreyling $M$, et al. Frontline therapy with rituximab added to the combination of cyclophosphamide, doxorubicin, vincristine, and prednisone (CHOP) significantly improves the outcome for patients with advanced-stage follicular lymphoma compared with therapy with $\mathrm{CHOP}$ alone: results of a prospective randomized study of the German LowGrade Lymphoma Study Group. Blood 2005;106:3725-32.

9. Marcus R, Imrie K, Belch A, et al. CVP chemotherapy plus rituximab compared with CVP as first-line treatment for advanced follicular lymphoma. Blood 2005;105:1417-23.

10. Herold $M$, Haas $A$, Srock $S$, et al. Rituximab added to first-line mitoxantrone, chlorambucil, and prednisolone chemotherapy followed by interferon maintenance prolongs survival in patients with advanced follicular lymphoma: an East German Study Group Hematology and Oncology Study. J Clin Oncol 2007;25:1986-92.

11. Salles G, Mounier N, de Guibert $S$, et al. Rituximab combined with chemotherapy and interferon in follicular lymphoma patients: results of the GELA-GOELAMS FL2000 study. Blood 2008;112:4824-31.

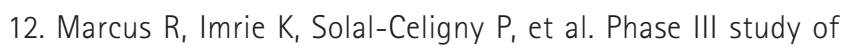
R-CVP compared with cyclophosphamide, vincristine, and prednisone alone in patients with previously untreated advanced follicular lymphoma. J Clin Oncol 2008;26:4579-86.

13. Russo AL, Chen $Y H$, Martin NE, et al. Low-dose involvedfield radiation in the treatment of non-hodgkin lymphoma: predictors of response and treatment failure. Int J Radiat Oncol Biol Phys 2013;86:121-7.

14. Hoskin PJ, Kirkwood AA, Popova B, et al. 4 Gy versus $24 \mathrm{~Gy}$ radiotherapy for patients with indolent lymphoma (FORT): a randomised phase 3 non-inferiority trial. Lancet Oncol 2014; 15:457-63.

15. Federico $M$, Bellei $M$, Marcheselli $L$, et al. Follicular lymphoma international prognostic index 2: a new prognostic index for follicular lymphoma developed by the international follicular Iymphoma prognostic factor project. J Clin Oncol 2009;27: 4555-62.

16. Bastion $Y$, Berger $F$, Bryon PA, Felman $P$, Ffrench $M$, Coiffier $B$. Follicular lymphomas: assessment of prognostic factors in 127 patients followed for 10 years. Ann Oncol 1991;2 Suppl 2:1239.

17. Leonard RC, Hayward RL, Prescott RJ, Wang JX. The identification of discrete prognostic groups in low grade nonHodgkin's lymphoma: the Scotland and Newcastle Lymphoma Group Therapy Working Party. Ann Oncol 1991;2:655-62.

18. Decaudin $D$, Lepage $E$, Brousse $N$, et al. Low-grade stage IIIIV follicular lymphoma: multivariate analysis of prognostic factors in 484 patients: a study of the groupe d'Etude des lymphomes de l'Adulte. J Clin Oncol 1999;17:2499-505.

19. Federico M, Vitolo U, Zinzani PL, et al. Prognosis of follicular Iymphoma: a predictive model based on a retrospective 
analysis of 987 cases. Intergruppo Italiano Linfomi. Blood 2000;95:783-9.

20. Solal-Celigny, Roy $P$, Colombat $P$, et al. Follicular lymphoma international prognostic index. Blood 2004;104:1258-65.

21. Federico M, Guglielmi $C$, Luminari $S$, et al. Prognostic relevance of serum beta2 microglobulin in patients with follicular Iymphoma treated with anthracycline-containing regimens: a GISL study. Haematologica 2007;92:1482-8.

22. Frank C, Zierhut D, Schulz-Ertner D, Wannenmacher M. Centroblastic-centrocytic non-Hodgkin's lymphoma stage I-III: patterns of failure following radiotherapy. Strahlenther Onkol 2001;177:597-603.

23. Ha CS, Kong JS, Tucker SL, et al. Central lymphatic irradiation for stage I-III follicular lymphoma: report from a singleinstitutional prospective study. Int J Radiat Oncol Biol Phys 2003;57:316-20.

24. Ahmed N, Owen TE, Rubinger M, et al. Early stage W.H.O. grade | and || follicular lymphoma treated with radiation therapy alone. PLoS One 2013;8:e65156.

25. Hainsworth JD, Litchy S, Burris HA 3rd, et al. Rituximab as first-line and maintenance therapy for patients with indolent non-hodgkin's lymphoma. J Clin Oncol 2002;20:4261-7.

26. Bachy $E_{1}$ Houot $R$, Morschhauser $F$, et al. Long-term follow up of the FL2000 study comparing CHVP-interferon to CHVP- interferon plus rituximab in follicular lymphoma. Haematologica 2013;98:1107-14.

27. Federico $M$, Luminari $S$, Dondi $A$, et al. R-CVP versus R-CHOP versus $\mathrm{R}-\mathrm{FM}$ for the initial treatment of patients with advanced-stage follicular lymphoma: results of the FOLLO5 trial conducted by the Fondazione Italiana Linfomi. J Clin Oncol 2013;31:1506-13.

28. Rummel MJ, Niederle N, Maschmeyer G, et al. Bendamustine plus rituximab versus $\mathrm{CHOP}$ plus rituximab as first-line treatment for patients with indolent and mantle-cell Iymphomas: an open-label, multicentre, randomised, phase 3 non-inferiority trial. Lancet 2013;381:1203-10.

29. Flinn IW, van der Jagt $R, K a h l B S$, et al. Randomized trial of bendamustine-rituximab or R-CHOP/R-CVP in first-line treatment of indolent NHL or MCL: the BRIGHT study. Blood 2014;123:2944-52.

30. De Los Santos JF, Mendenhall NP, Lynch JW Jr. Is comprehensive lymphatic irradiation for low-grade non-Hodgkin's Iymphoma curative therapy? Long-term experience at a single institution. Int J Radiat Oncol Biol Phys 1997;38:3-8.

31. McClanahan $F$, Hielscher $T$, Rieger $M$, et al. Clinical outcome of patients with follicular lymphoma and bulky disease after rituximab-CHOP immunochemotherapy with and without consolidating radiotherapy. Eur J Haematol 2010;85:11-9. 